\title{
Public and Population Health Informatics: The Bridging of Big Data to Benefit Communities
}

\author{
Roland Gamache ${ }^{1,2}$, Hadi Kharrazi ${ }^{1,3}$, Jonathan P. Weiner ${ }^{1}$ \\ 1 Center for Population Health Information Technology, Johns Hopkins Bloomberg School of Public \\ Health, Baltimore, USA \\ 2 Gamache Consulting, Bethesda, USA \\ 3 Division of Health Sciences and Informatics, Johns Hopkins School of Medicine, Baltimore, USA
}

\begin{abstract}
Summary
Objective: To summarize the recent public and population health informatics literature with a focus on the synergistic "bridging" of electronic data to benefit communities and other populations. Methods: The review was primarily driven by a search of the literature from July 1, 2016 to September 30, 2017. The search included articles indexed in PubMed using subject headings with (MeSH) keywords "public health informatics" and "social determinants of health". The "social determinants of health" search was refined to include articles that contained the keywords "public health", "population health" or "surveillance".

Results: Several categories were observed in the review focusing on public health's socio-technical infrastructure: evaluation of surveillance practices, surveillance methods, interoperable health information infrastructure, mobile health, social media, and population health. Common trends discussing socio-technical infrastructure included big data platforms, social determinants of health, geographical information systems, novel data sources, and new visualization techniques. A common thread connected these categories of workforce, governance, and sustainability: using clinical resources and data to bridge public and population health.

Conclusions: Both medical care providers and public health agencies are increasingly using informatics and big data tools to create and share digital information. The intent of this "bridging" is to proactively identify, monitor, and improve a range of medical, environmental, and social factors relevant to the health of communities. These efforts show a significant growth in a range of population health-centric information exchange and analytics activities.
\end{abstract}

\section{Keywords}

Public health informatics; population health; social determinants of health; surveillance

Yearb Med Inform 2018:199-206 http://dx.doi.org/10.1055/s-0038-1667081

\section{Introduction}

Over the last several decades, powerful forces have motivated the collection of health-related digital data in the United States (US) and around the world. The current US healthcare's electronic data infrastructure has been designed primarily to meet the needs of medical providers' health insurance billing, internal organizational management, and regulatory reporting. Public health officials have mainly focused on the construction of data systems to document communicable diseases and other reportable events [1]. The disconnection of data management aims across the healthcare continuum has led to silos of isolated electronic health data [2]. Attempts at integrating these data sources for the purpose of improving the health of a community or a defined population has, for the most part, been viewed as a "secondary" application among health providers that has not risen to a priority level [3]. Indeed, with the exception of a few highly integrated medical delivery systems, until the last few years, most clinicians in the US had little interest and even fewer incentives to advance population health applications by linking their data across these silos [4].

One of the first attempts at integrating the clinical and public data to advance population health relates to the implementation of electronic health records (EHR)-based surveillance efforts in a few, limited jurisdictions [5]. In the US, however, this trend has recently started to shift in a new direction, reinforced and supported by changes in policy, alignment of incentives, and the wider adoption of data sharing protocols and infrastructure [2]. The so called "value-based" policies of commercial and government insurance com- panies have begun to shift the focus of clinical organizations from individual patient visits to managing larger populations and improving their overall health outcomes while maintaining cost efficiency $[6,7]$. Some recent state and federal policies have introduced increased financial risk-sharing and fixed budgets for provider organizations, hence pushing clinicians to apply their digital assets to address social determinants of health (SDH), defined as gaps in care and other non-clinical factors that may lead to costly tertiary interventions for which they receive no reimbursement $[8$, 9]. These recent powerful trends have served to align medical and public health aims at a level never seen before. A number of health information technology (IT) solutions, such as health information exchanges (HIEs) and population or community level analytics have propelled the collaboration of health systems and public health departments to better manage their overlapping "community" denominators to integrate across many different digital silos [10].

The alignment of policies and the new fiscal incentives facing the powerful hospital and physician provider sectors in the US whose overall budgets dwarf that of the US public health sector by a factor of over 25 [11] - have led to an unprecedented sharing of data, information, and knowledge with the ultimate goal of improving the overall health of communities [12]. Given these fast-paced and emergent trends, this year's review focuses on the synergistic "bridging" of electronic data to reshape public and population health informatics efforts in the US. In addition, we identify selected international references from European and other high-income nations that are noteworthy and within our scope. While the US context is 
somewhat unique, we attempt to make our discussion and implications relevant to the global health informatics community.

\section{Methods}

This work has primarily been driven by a search of the current literature spanning from July 1, 2016 to September 30, 2017. A number of articles published after this timeframe that were highlighted at several scientific venues (e.g., 2017 AMIA Symposium [13]) were also included. The initial literature search included articles indexed in PubMed using the Subject Headings (MeSH) keywords "public health informatics" and "social determinants of health". The "social determinants of health" search was refined to only include articles that contained the keywords "public health", "population health" or "surveillance".

There were a handful of key activities that led to the authors' choice of current public health trends. One of the main drivers was the conceptual development of the focus of activities for "Public Health 3.0". Public Health 3.0 calls for a cross-sector collaboration that directly affects the social determinants of health [14]. Another guiding factor was the public health support for the "Digital Bridge" project, which is a partnership aiming at ensuring health in the US by establishing bidirectional data exchanges between health care and public health [15]. Lastly, another source of direction was the Public Health Informatics Policy Summit in 2017, convened by the (American) Association of State and Territorial Health Officials (ASTHO). This event highlighted several emerging public health activities in the near future, with two over-arching areas on the increased need of social determinants of health for public health efforts, and the need for policies to define a process for public health officers to assume a larger role for SDH interventions in their communities.

Using the MeSH terms and constraints described above, 897 articles were returned using PubMed. Articles that were primarily focused on clinical delivery, systematic reviews, commentaries, letters, opinions, white papers, and study protocols were excluded, leading to 134 articles. These remaining articles were grouped into seven categories based on keywords in the PubMed index. The included general informatics/data science focus areas were: public health 3.0, big data, population health, SDH, surveillance, privacy, confidentiality and transparency of data, and workforce development and training. These themes were based on comments from public and population health experts from several key informatics meetings held during the year. After reviewing these articles, we determined that 51 of them represented noteworthy or "trending" efforts, and thus we selected them for discussion in this manuscript.

A generally accepted definition of public health informatics is: "the systematic application of information, computer science, and technology to public health practice, research, and learning" [16]. Population health comprises organized activities for assessing and improving the health and well being of a defined population. The target "population" can be a specific geographic community or region, or it may be a defined group of individuals such as enrollees of a health plan, persons residing in a provider's catchment area, or an aggregation of individuals with special needs [12]. Population health informatics addresses the information technology and analytic needs of groups and organizations responsible for the health management of defined populations. We adopted these definitionsto build on the conceptual framework that was developed and applied in previous reports in this series [5 17]. For this article, we updated the previous framework to reflect the current trend of expanded integration of SDH factors into the public health strategic partnerships, and the IT infrastructure supporting surveillance and analytics [18] (Figure 1). We modified the previous framework to adjust for this trend, and we deleted the global health (i.e., in low income settings) present in past frameworks, as that topic, while very important, was out of scope. We also acknowledge that integrating social services/social welfare informatics with health data may be considered standard - and not a "new" trend - in some European nations, but in the US, the topic of digital solutions to better address "social determinants" within the population health context is considered novel in many settings and thus is a special focus of this article and accordingly, the unifying framework presented in Figure 1.

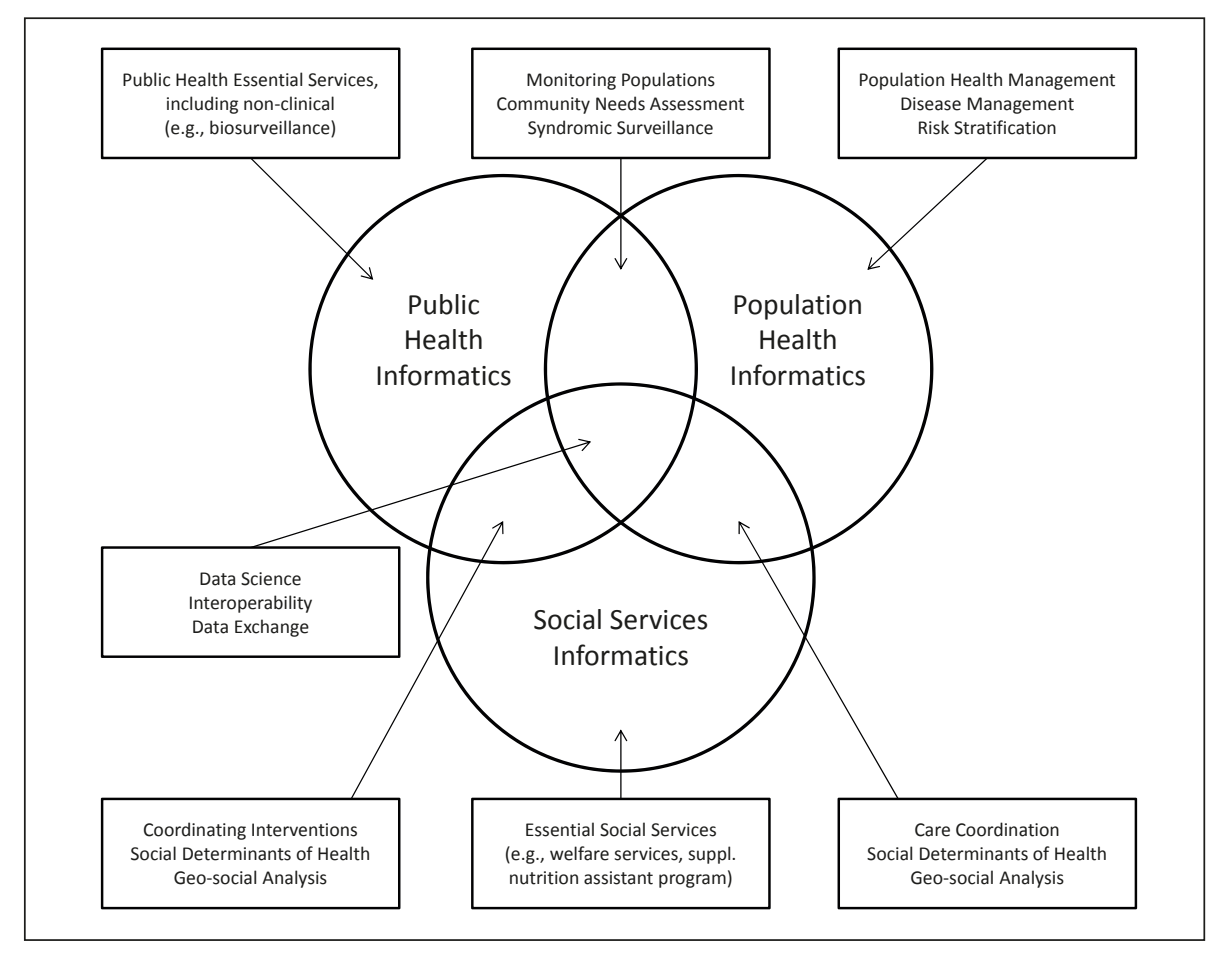

Fig. 1 Overlapping domains related to Public Health, Population Health, and Social Services Informatics 


\section{Results}

Though not an exhaustive systematic review, using the structured process described above to identify in-scope articles, we present an overview and a discussion of major trends and topics that resonate with public and population health informatics professionals.

\section{(1) Socio-Technical Infrastructure and Research}

The definition of "Big Data" seems to vary depending on the author and context [19]. One common area of the public health discussion involving Big Data is the growing variety of data sources and types of available health-related data. The sources ranged from drug discovery and personal health [20] to the application of data mining and analytical techniques to extract information and knowledge from multiple and diverse datasets to examine the opportunities to improve event detection for injury surveillance [21]. The National Institute of Standards and Technology provides a definition of Big Data that overlaps with the public health use of this term. It includes many of the common themes of the socio-technical infrastructure, in particular the increased use of unstructured data, the impact on society, more data than before, and the loss of privacy [22].

\section{(1-a) Evaluation of Surveillance Practice}

The inclusion of SDH in public health surveillance and practice has seen considerable innovation. Some of the SDH factors that have been included in public health data systems are summarized in a discussion of metrics to assess neighborhood walkability and associated walkability that were linked to increased physical activity [23]. As the population living in urban areas is increasing rapidly, our understanding of SDH and city-related livability factors will be important, particularly for low and middle income countries [24].

US public health agencies are increasingly developing agreements to use social services data collected by government agencies, outside of public health, to help track areas of risk in the population [25]. However, US public health agencies need to work actively to catch up with results achieved by other countries, such as Scotland, that have linked multiple agency sources to access the health of children that were in the responsibility of the jurisdiction [26]. However, it is clear that more research is required before applying the same tools in resource-constrained countries [24].

Communicable disease surveillance and management has been one of the main efforts of public health for decades; however, there are still innovative practice enhancements to improve the timeliness and quality of these activities while reducing the burden on physicians [27, 28]. Recent publications have also focused on using new data streams from clinical health systems as a substitute to survey data to monitor chronic diseases in the community [29]. A growing trend among public health agencies is the use of near real-time syndromic surveillance systems to aid in public health decisions. Public health agencies have used these systems to monitor the impact of potential environmental triggers that may increase chronic disease visits to emergency departments, to target prevention programs and strategies, to develop epidemiology profiles for opioid use and other adverse drug events, and to monitor emerging disease threats such as Ebola [30, 31].

\section{(1-b) Surveillance Methods}

With increased accessibility to health delivery data by both public health and health care professionals, there has been an increase in the use of spatial analytics to monitor and understand the spread of disease and outbreak management responses [32]. For example, geographic information systems (GIS) allow communities to better utilize public health resources by targeting a community immunization response strategy to outbreak clusters [33], although selection bias could be an issue, particularly for those from rural settings, certain ethnic or racial groups, and those with lower socio-economic status [34]. In addition, GIS surveillance can be assisted by social media. One study used Twitter feeds within 31 US cities for purposes of real time influenza surveillance. Interestingly, the study noted that a good understanding of the local culture is required to fully assess such models [35]. New analytical approaches such as Hadoop-GIS in combination with multiple data sources such as Topologically Integrated Geographic Encoding and Referencing (TIGER) and Census data were used in combination with traditional public health data such as the American Community Survey [36], to improve community surveillance and outreach.

One encouraging area of improvement in public health surveillance has been the adoption of an algorithm to automatically fill in all of the fields of the reportable disease forms directly from data available in the EHR [37]. These "auto filled" forms reduced the reporting burden on clinicians, decreased the time of the initial report by 2.7 days, and reduced the time to close a case by 0.2 day.

\section{(1-c) Interoperable Health Information Infrastructure}

The use of other digital tools was also employed in several epidemiologic studies. One paper examined the use of REDCap, a secure web application for data collection and surveys (https://project-redcap.org) as a data collection tool that was used as a follow-up to a birth cohort study [38]. These tools shortened the time to collect and validate the data from several months to two weeks.

\section{(1-d) mHealth, Social Media, and Visualization}

Although telehealth may appear primarily as a clinical service enhancement, it can serve as a safety net provider for vulnerable populations, and as such, it is an essential service of many public health agencies in the US. A study in South Africa rated the quality of images using smart phones and tablets by emergency room physicians as good as or better than a standard computer monitor to review images [39]. The analysis from this study indicates a benefit for mHealth tools in health care delivery. This clinical study demonstrates the quality of information that may be used to provide access to services and specialists in areas with few available physicians.

There were two studies in London (United Kingdom) that looked at the use of text messaging as a method to increase screening rates within a population $[40,41]$. The effectiveness of the intervention is dependent on the screening test and the target population. Advanced semantic and linguistic analysis 
methods have also enabled researchers to effectively identify associated topics in these social media data. These techniques were effectively used to find common topics used in Twitter posts that discuss obesity, diet, exercise, and diabetes [42].

New tools in data visualization and their application in health care and public health have generated a great deal of interest in the last few years. The Pan American Health Organization (PAHO) and the Centers for Disease Control and Prevention (CDC) have developed interactive visualization platforms to aid in injury prevention in the US [43].

\section{(1-e) Population Health}

Population health studies over extended time frames face concerns regarding the integrity of the data due to variation in data standards, data collection protocols, and measurement tools or devices over the period in question. A description of a large cohort in France highlights the informatics challenges associated with maintaining such a robust population level data system [44].

\section{(2) Workforce Development}

A number of publications pointed out that public health agencies realize that they will need to continually update competencies as these new data sources evolve. However, the areas of development may vary depending on the size of the health department [45]. For example, based on a survey from the National Association of City and Country Health Officials (NACCHO) on workforce development, the use of statistical and analytical software was a high priority, but the indicated need for this training varied from $35.2 \%$ for health department serving populations of less than 50,000 to $71.7 \%$ for health departments serving populations over 500,000 . In fact, these self-identified development needs tend to correlate with the informatics activities typically performed at these departments [46]. Moreover, there is a call for epidemiologic training "to link more strongly with emerging technologies and to acknowledge key societal transformations [47].

\section{(3) Governance, Ethics, and Confidentiality}

One key priority area for the public health community is the ethical application of digital data for public health and popula- tion level research [48]. Public health has always had the responsibility of balancing potential health benefits and dangers within a community with personal choices and welfare of individuals that comprise the community. There is now a digital version of this balancing act; that is, achieving equilibrium between complete data to mitigate community dangers and promote collective benefit, while mitigating risk to individual privacy and confidentiality [49]. To help avoid these person-level risks, there is growing interest in both data governance and innovative technology. To gain a better understanding of how consumers feel about the balance between community welfare versus individual privacy, recent studies have examined individuals' levels of support for government and others accessing patient records for community level activities, including public health [50].

\section{(4) Sustainability}

In the recent literature, two areas were discussed within the topic of sustainability of digital initiatives [51]. The first area identified was the importance of strategic partnerships within the community in order to achieve public and population health objectives using informatics tools [18]. These partnerships should not only lay out digitally-supported plans for the improvement of the health status of the community, but they should also encourage stakeholders from outside traditional public health agencies to understand and to be involved in these processes [52]. One emerging effort in this partnership is the use of epidemiologic profiles used by health care providers to address risky health behaviors at a time or manner that increases the probability of the individual making a positive change in behavior. Although these may not be a direct public health intervention, the developed profiles result from the data obtained from public health and health care delivery. These profiles are used to develop automated clinical reminders to alert physicians to review these risky behaviors with the patient during a clinical encounter. The effectiveness and description of the use of these reminders to help patients stop smoking is discussed in this paper [53].
The second area of discussion concerned a stable funding source providing fundamental public health services [54]. New models to provide for public health fundamental services will include both population health activities and governmental public health services [55].

\section{Discussion}

This article reviewed the latest developments and trends of public and population health informatics. Slightly different from previous updates in this yearbook monograph series [5, 17], in addition to highlighting key observations and findings that cited authors have written about in the recent 2016-2017 literature, this article also discusses key trends based on several recent US public health informatics policy meetings. The intent of these workshops was to assess the future informatics needs for public health given new methods of digital data gathering and the potential impact of these changes for public health, particularly for surveillance and epidemiology activities. Another change from the reviews of previous years is the increased acknowledgement within the public health informatics community of the heightened value of digitally capturing SDH risk factors and their complex interaction with public health and clinical outcomes at both the community and individual levels [56, 57].

A summation and synthesis of the recent trends of public and population health informatics that we have identified above is organized into three thematic areas: the newly emerging public health informatics vision and infrastructure; the alignment of informatics aims, goals, and outcomes across the oftentimes separate fields of public health and population health; and the increased incorporation by both public and population health informatics professionals of SDH data.

\section{(1) Public Health Informatics 3.0}

Public health leadership has a unique opportunity to expand the business case for public health and population health information systems [52]. This additional authority has not been part of a new legal 
mandate, but the result of the availability of new sources of data and the rate at which these new and existing data are now being sent to public health agencies [55]. Access to timely and reliable health data sources has decreased the need of public health to aggregate, clean, and validate this data de novo. Therefore, these data are now a valuable resource for both public health and the providers of the data. The desire of public health partners to use these data has increased the importance of firming relationships with historical and new public health partners [58]. Since this timely and reliable source of data is now available to public health partners, the strategic efforts and direction for improving the health of the community is now shared, to a larger extent, with community partners including payers, accountable care organizations, and health care delivery systems [59].

Additionally, as the metrics for SDH continue to be accepted and validated, the use of these social metrics is expanding the need for data collection and analysis outside traditional clinical settings (e.g., within human service agencies) and requires new partners in the clinical space that have historically partnered with public health agencies [60]. Helping to facilitate this digital linkage between social and provider organizations could be a role for public health agencies. Also, it will be important for public health agencies to ensure that digital systems capture SDH factors quite broadly and not just those linked to "medical" conditions. For example, SDH data systems should define the community environment in this context, including digital metrics describing features such as crime, housing stock, social networking, measures of pollution, and toxic substance exposure.

Along with the new informatics leadership roles for public health, there must be a workforce available to manage and analyze the data shared across the wider range of partners. It is important for public health staff to have a thorough understanding of informatics, data flows, data collection processes, and the use of data at the public health agency in order to work productively with a larger and more diverse group of community partners, including medical providers and social service agencies.
This complex population health informatics infrastructure may be viewed as a network of nodes within the community, each with a specialized set of data and knowledge expertise [61]. To be valuable to all partners, the electronic data available to the growing circle of stakeholders must provide reliable, timely, and actionable data that will inform and add value to the component groups providing the data. This data sharing network will need to be transparent on how data are collated, protected, and shared with members of consortia and others. And they must find the wherewithal to provide state-of-the art analytics and visualization tools focusing on community, population, and social welfare points of view, something that so far has been challenging to do, when compared to the better funded fields of clinical and healthcare management data sciences and informatics.

\section{(2) Alignment of Population and Public Health Informatics'Aims and Outcomes}

In the recent years, major efforts by the US Medicare and Medicaid Services as well as private insurers have offered strong fiscal incentives for providers to adopt so called "value-based" delivery models, such as accountable care organizations and patient-centered medical homes, where clinicians receive higher payment for delivering better and more efficient care [6]. Specifically, these models provide reimbursement for the improved health outcomes of a defined patient population rather than individual visits and services. This shift in the reimbursement models, which is enacted and supported by various policies [7], has motivated providers to better manage their entire patient population while controlling the overall cost of care. Under some of these models, value-based providers may receive a global budget for their assigned population, thus a reduced rate of utilization translates into larger shared-savings for both providers and payers. The latter has shifted the focus of value-based providers into prevention efforts (hence lower utilization) rather than costly treatment interventions, which aligns well with the aims of public health departments on reducing preventable diseases in large populations.

As of 2017, in certain US states such as Maryland, health care financing is substantially moving towards global budgets based on the size and characteristics of the population living in a catchment area, rather than based only on those who present themselves for services [62]. This effort will be the first time that a large component of an entire state's health system's budget is based on a population of people who may not utilize the health system at all. In fact, these changes will effectively turn health systems into entities that are responsible for the health of a population living in a geographical area thus stimulating collaboration with local health departments. These incremental changes will eventually align the aims and goals of health systems and health departments to improve the health outcomes of their ever increasing and overlapping populations. National efforts on closing the gap between clinical care and community services (e.g., accountable health communities) also support the alignment of population health and public health aims [63].

In response to the alignment of aims and goals, the informatics community has also started to align data sources, infrastructure and data sharing among population health, public health, and to some extent social services entities. In the US, EHR adoption has skyrocketed among providers, giving them the opportunity to collect data on large patient populations. The expanded data exchange standard adoptions have increased the possibility of sharing data among providers and public health departments. These technological drivers have created a unique opportunity to utilize each side's IT infrastructure to boost the aligned aims and outcomes of both groups. For example, a number of studies included in this review depicted that data collected by a large health system or HIE has been used to study the prevalence of a specific chronic disease in a given geography, a task that has been traditionally accomplished by health departments using exhaustive survey methods for public health needs assessment and monitoring purposes [64-67]. Moreover, data collected by public health departments are a key source of data required to calculate population-level health measures that will eventually be used to determine global budgets for medical care delivery systems and to assess whether they reach their community health targets in order to achieve substantial financial incentives [68]. And finally, the sharing of SDH data across public health, 
population health, and social services is the prime example of how the landscape of public and population health informatics practice is going to change and potentially converge in the coming years [8].

\section{(3) Social Determinants of Health}

Recently, the US health informatics landscape has seen an increased emphasis on data integration across the previously separate clinical, management, insurance, and public health data silos. With this shift in thinking, there is also an increased acknowledgment of the importance of considering a broad array of "non-medical" factors generally termed as social determinants of health. Along with this recognition has come a surge of interest in data, metrics, IT systems, and advanced analytics that allow for the appropriate inclusion of SDH information into a broad array of health and medical applications and use cases [69-71].

Internationally, and among social and public health scientists in the US, the importance of integrating social factors into the medical care delivery process - strategically, programmatically, and analytically - is hardly news. But in the commercially dominated US health care delivery and financing system, that has not been the case. Today in the US, there has never been wider interest in medical/ SDH integration among rank and file clinicians, health insurance and delivery system administrators, and the IT vendors that serve them. Accordingly, not only are more social factors, such as those related to employment, housing, language barriers, captured and integrated into existing medical and administrative data systems, but new types of data from previously untapped sources are now increasingly being combined with medical informatics systems.

These non-medical data, which include consumer/client level data derived from IT systems of human service government and non-profit agencies, such as those providing housing and food assistance and support for domestic violence, as well as "consumer" level corporate data (e.g., from marketing firms or banks) were previously used only for commercial purposes. Additionally, large amounts of government (e.g., US Census) and private data at the neighborhood level are being collated and linked with the established health care and medical data to help provide context on important geographically linked factors that have an impact on health. Some examples of these geo-linked data include environmental risks and hazards, neighborhood crime and safety, availability and quality of housing stock, ease of transportation, and availability of healthy food.

\section{Limitations}

This review focused on recently published literature primarily related to the areas of public and population health with an informatics theme, so there may be pertinent articles from the health care delivery field that were not included. Although articles from other countries were included in our findings, these results did not include articles that were not indexed in PubMed or not written in English. An other shortcoming of this review was that findings from the global health community were not included. Several articles were included in our findings that did not have keywords or an abstract included with the citation. These articles were manually added to the topic areas based on a manual review of the papers. Staff in the public health field are usually focused on public health practice and these results may only appear in white papers or policy statements and are not indexed in PubMed. Finally, most peer-reviewed articles focus on the positive results that are achieved. Due to this publication bias, many informative studies that have a negative outcome may not be published. This paper, as in previous editions, did not include search terms for "epidemiology" and "informatics". These search terms retrieved thousands of journal articles with most not pertinent to this review. If additional terms were added to reduce the list, by adding public health or population health, these articles would have been included in the original search parameters.

\section{Conclusion and Future Directions}

Both medical care providers and public health agencies are increasingly using informatics and various tools to create and share digital information both within and across sectors. Their intent is to proactively identify, monitor, and if possible, alter a wide range of medical, environmental, and social factors that are relevant to the health of communities or other pre-defined patient cohorts. These efforts will, over the coming decade, result in a very significant growth in a wide range of population health-centric information exchange and analytics activities. All this research will digitally embrace a multi-faceted array of non-clinical risk factors that have the potential to prevent negative health outcomes and support personal and community wellbeing.

Thanks to new financial and regulatory infrastructures, as well as shifts in the care paradigm, there has been increased attention among US medical care providers in population-based prevention activities intended to reduce readmissions and other avoidable (and expensive) medical events. These powerful incentives are catalyzing the formation of partnerships between public health agencies, social service agencies, and medical care providers whose target populations overlap. These cross-sector collaborations are slowly leading to an increased bridging of clinical, administrative, public health, and social service data systems, which may ultimately lead to a fully integrated population health informatics infrastructure. A common tool for this "Health 3.0" integration is the expanded focus on social and environmental determinants that previously were not well addressed by the medical sector, digitally or otherwise. While evidence is starting to be captured, a full assessment of the impact of such SDH-focused, informatics-mediated, interventions awaits further scientific evaluations [72].

In the US, like in other industrialized counties, there is a need to bridge the considerable divide between medical and public health agencies. Recent advances in population health informatics as described in this review suggest that expanded sources of big data and the increased availability of sophisticated health IT tools serve to effectively increase the integration and coordination of functions of these two sectors to the benefit of their communities. Furthermore, as many parties have been reminding us for decades, acknowledging, measuring, and addressing 
social and other "non-medical" determinants of health, and then digitally integrating all these data into the mainstream medical care context will be essential to achieving true health and wellbeing among the populations living in our neighborhoods, regions, and nations.

\section{References}

1. Baker EL, Fond M, Hale P, Cook J. What Is "Informatics"? J Public Health Manag Pract 2016;22(4):420-3.

2. Adler-Milstein J, Embi PJ, Middleton B, Sarkar IN, Smith J. Crossing the health IT chasm: considerations and policy recommendations to overcome current challenges and enable value-based care. J Am Med Inform Assoc 2017;24(5):1036-43.

3. Eggleston EM, Finkelstein JA. Finding the role of health care in population health. JAMA 2014;311(8):797-8.

4. Kharrazi H, Weiner JP. IT-enabled Community Health Interventions: Challenges, Opportunities, and Future Directions. Egems 2014;2(3):1117.

5. Dixon BE, Kharrazi H, Lehmann HP. Public Health and Epidemiology Informatics: Recent Research and Trends in the United States. Yearb Med Inform 2015;10(1):199-206.

6. CMS. Core Measures. Secondary Core Measures. https://www.cms.gov/Medicare/Quality-Initiatives-Patient-Assessment-Instruments/QualityMeasures/Core-Measures.html.

7. CMS. MACRA-MIPS-and-APMs/MACRAMIPS-and-APMs. Secondary MACRA-MIPSand-APMs/MACRA-MIPS-and-APMs. https:// www.cms.gov/Medicare/Quality-Initiatives-Patient-Assessment-Instruments/Value-Based-Programs/MACRA-MIPS-and-APMs/MACRAMIPS-and-APMs.html.

8. Ash AS, Mick EO, Ellis RP, Kiefe CI, Allison JJ, Clark MA. Social Determinants of Health in Managed Care Payment Formulas. JAMA Intern Med 2017;177(10):1424-30.

9. Patel A, Rajkumar R, Colmers JM, Kinzer D, Conway PH, Sharfstein JM. Maryland's Global Hospital Budgets--Preliminary Results from an All-Payer Model. N Engl J Med 2015;373(20):1899-901.

10. Kharrazi H, Horrocks D, Weiner J. Use of HIEs for Value-Based Care Delivery: A Case Study of Maryland's HIE. Health Information Exchange: Navigating and Managing a Network of Health Information Systems. Elsevier Inc.; 2016. p. 313-32.

11. Keehan SP, Stone DA, Poisal JA, Cuckler GA, Sisko AM, Smith SD, et al. National Health Expenditure Projections, 2016-25: Price Increases, Aging Push Sector To 20 Percent Of Economy. Health Aff (Millwood) 2017;36(3):553-63.

12. Kharrazi H, Lasser EC, Yasnoff WA, Loonsk J, Advani A, Lehmann HP, et al. A proposed national research and development agenda for population health informatics: summary recommendations from a national expert workshop. J Am Med Inform
Assoc 2017;24(1):2-12.

13. AMIA. AMIA 2017 Annual Symposium. Secondary AMIA 2017 Annual Symposium 2017. https:// www.amia.org/amia2017.

14. DeSalvo KB, O`Carroll PW, Koo D, Auerbach JM, Monroe JA. Public Health 3.0: Time for an Upgrade. Am J Public Health 2016;106(4):621-2.

15. Digital Bridge. Secondary Digital Bridge 2017. http://www.digitalbridge.us/about/.

16. Yasnoff WA, O'Carroll PW, Koo D, Linkins RW, Kilbourne EM. Public health informatics: improving and transforming public health in the information age. J Public Health Manag Pract 2000;6(6):67-75.

17. Massoudi BL, Chester KG. Public Health, Population Health, and Epidemiology Informatics: Recent Research and Trends in the United States. Yearb Med Inform 2017;26(1):241-47.

18. DeSalvo KB, Wang YC, Harris A, Auerbach J, Koo D, O'Carroll P. Public Health 3.0: A Call to Action for Public Health to Meet the Challenges of the 21st Century. Prev Chronic Dis 2017;14:E78.

19. Ward SJ, Barker A. Undefined By Data: A Survey of Big Data Definitions. 201320 Sep 2013.

20. Leyens L, Reumann M, Malats N, Brand A. Use of big data for drug development and for public and personal health and care. Genet Epidemiol 2017;41(1):51-60.

21. Gunn JE, Shah SN. Big data and opportunities for injury surveillance. Inj Prev 2016;22 Suppl 1:i3-5.

22 Big Data Definitions. Secondary Big Data Definitions. https:/bigdatawg.nist.gov.

23. Rundle AG, Sheehan DM, Quinn JW, Bartley K, Eisenhower D, Bader MMD, et al. Using GPS Data to Study Neighborhood Walkability and Physical Activity. Am J Prev Med 2016;50(3):e65-e72.

24. Prasad A, Gray CB, Ross A, Kano M. Metrics in Urban Health: Current Developments and Future Prospects. Annu Rev Public Health 2016;37:113-33

25. Beck A, Davidson AJ, Xu S, Josh Durfee M, Oronce CIA, Steiner JF, et al. A Multilevel Analysis of Individual, Health System, and Neighborhood Factors Associated with Depression within a Large Metropolitan Area. J Urban Health 2017.

26. Clark D, King A, Sharpe K, Connelly G, Elliott L, Macpherson LMD, et al. Linking routinely collected social work, education and health data to enable monitoring of the health and health care of school-aged children in state care ('looked after children') in Scotland: a national demonstration project. Public Health 2017;150:101-11.

27. Dixon BE, Zhang Z, Lai PTS, Kirbiyik U, Williams $\mathrm{J}$, Hills R, et al. Completeness and timeliness of notifiable disease reporting: a comparison of laboratory and provider reports submitted to a large county health department. BMC Med Inform Decis Mak 2017;17(1):87.

28. Revere D, Hills RH, Dixon BE, Gibson PJ, Grannis SJ. Notifiable condition reporting practices: implications for public health agency participation in a health information exchange. BMC Public Health 2017;17(1):247.

29. Klompas M, Cocoros NM, Menchaca JT, Erani D, Hafer E, Herrick B, et al. State and Local Chronic Disease Surveillance Using Electronic Health Record Systems. Am J Public Health 2017;107(9):1406-12.
30. Lall R, Abdelnabi J, Ngai S, Parton HB, Saunders $\mathrm{K}$, Sell J, et al. Advancing the Use of Emergency Department Syndromic Surveillance Data, New York City, 2012-2016. Public Health Rep 2017;132(1_suppl):23S-30S.

31. Yoon PW, Ising AI, Gunn JE. Using Syndromic Surveillance for All-Hazards Public Health Surveillance: Successes, Challenges, and the Future. Public Health Rep 2017;132(1_suppl):3S-6S.

32. Ben Ramadan AA, Jackson-Thompson J, Boren SA. Geographic Information Systems: Usability, Perception, and Preferences of Public Health Professionals. Online J Public Health Inform 2017;9(2):e191.

33. Maravi ME, Snyder LE, McEwen LD, DeYoung K, Davidson AJ. Using Spatial Analysis to Inform Community Immunization Strategies. Biomed Inform Insights 2017;9:1178222617700626.

34. Ha S, Hu H, Mao L, Roussos-Ross D, Roth J, Xu $\mathrm{X}$. Potential selection bias associated with using geocoded birth records for epidemiologic research. Ann Epidemiol 2016;26(3):204-11.

35. Allen C, Tsou MH, Aslam A, Nagel A, Gawron JM. Applying GIS and Machine Learning Methods to Twitter Data for Multiscale Surveillance of Influenza. PLoS One 2016;11(7):e0157734.

36. Chen X, Wang F. Integrative Spatial Data Analytics for Public Health Studies of New York State. AMIA Annu Symp Proc 2016;2016:391-400.

37. Painter I, Revere D, Gibson PJ, Baseman J. Leveraging public health's participation in a Health Information Exchange to improve communicable disease reporting. Online J Public Health Inform 2017;9(2):e186.

38. Blumenberg C, Barros AJ. Electronic data collection in epidemiological research. The use of REDCap in the Pelotas birth cohorts. Applied clinical informatics 2016;7(3):672-81.

39. Boissin C, Blom L, Wallis L, Laflamme L. Image-based teleconsultation using smartphones or tablets: qualitative assessment of medical experts. Emerg Med J 2017;34(2):95-99.

40. Herrett E, Williamson E, van Staa T, Ranopa M, Free $\mathrm{C}$, Chadborn T, et al. Text messaging reminders for influenza vaccine in primary care: a cluster randomised controlled trial (TXT4FLUJAB). BMJ Open 2016;6(2):e010069.

41. Hirst Y, Skrobanski H, Kerrison RS, Kobayashi LC, Counsell N, Djedovic N, et al. Text-message Reminders in Colorectal Cancer Screening (TRICCS): a randomised controlled trial. Br J Cancer 2017;116(11):1408-14.

42. Karami A, Dahl AA, Turner-McGrievy G, Kharrazi $\mathrm{H}$, Shaw $\mathrm{G}$. Characterizing diabetes, diet, exercise, and obesity comments on Twitter. Int J Inf Manage 2018;38(1):1-6.

43. Martinez R, Ordunez P, Soliz PN, Ballesteros MF. Data visualisation in surveillance for injury prevention and control: conceptual bases and case studies. Inj Prev 2016;22 Suppl 1:i27-33.

44. Ruiz F, Goldberg M, Lemonnier S, Ozguler A, Boos E, Brigand A, et al. High quality standards for a large-scale prospective population-based observational cohort: Constances. BMC Public Health 2016;16(1):877.

45. Bakota E, Arnold R, Yang B. Investigating Informatics Activity, Control, and Training Needs in Large, Medium, and Small Health Departments. 
J Public Health Manag Pract 2016;22 Suppl 6, Public Health Informatics:S63-S68.

46. Drezner K, McKeown L, Shah GH. Assessing Skills and Capacity for Informatics: Activities Most Commonly Performed by or for Local Health Departments. J Public Health Manag Pract 2016;22 Suppl 6, Public Health Informatics:S51-S57.

47. Brownson RC, Samet JM, Bensyl DM. Applied epidemiology and public health: are we training the future generations appropriately? Ann Epidemiol 2017:27(2):77-82.

48. Salerno J, Knoppers BM, Lee LM, Hlaing WM, Goodman KW. Ethics, big data and computing in epidemiology and public health. Ann Epidemiol 2017;27(5):297-301.

49. Hawkes N. Public supports commercial access to patient records when public benefit is clear. BMJ 2016;352:i1414.

50. Martin EG, Begany GM. Opening government health data to the public: benefits, challenges, and lessons learned from early innovators. J Am Med Inform Assoc 2017;24(2):345-51.

51. Leider JP, Shah GH, Williams KS, Gupta A, Castrucci BC. Data, Staff, and Money: Leadership Reflections on the Future of Public Health Informatics. J Public Health Manag Pract 2017;23(3):302-10.

52. Baker EL, Brand W, Davidson A, LaVenture M, Singletary V, Smith P. Building the Business Case for Public Health Information Systems. J Public Health Manag Pract 2016;22(6):603-6.

53. Bae J, Ford EW, Kharrazi HHK, Huerta TR. Electronic medical record reminders and smoking cessation activities in primary care. Addict Behav 2017;77:203-09.

54. Phelps C, Madhavan G, Rappuoli R, Levin S, Shortliffe E, Colwell R. Strategic Planning in Population Health and Public Health Practice: A Call to Action for Higher Education. Milbank Q 2016;94(1):109-25.

55. DeSalvo K, Wang YC. Health Informatics in the Public Health 3.0 Era: Intelligence for the Chief Health Strategists. J Public Health Manag Pract
2016;22 Suppl 6, Public Health Informatics:S1-S2. 56. White JR, Berisha V, Lane K, Ménager H, Gettel A, Braun CR, et al. Evaluation of a Novel Syndromic Surveillance Query for Heat-Related Illness Using Hospital Data From Maricopa County, Arizona, 2015. Public Health Rep 2017;132(1_sup$\mathrm{pl}): 31 \mathrm{~S}-39 \mathrm{~S}$

57. Koo D, O'Carroll PW, Harris A, DeSalvo KB. An Environmental Scan of Recent Initiatives Incorporating Social Determinants in Public Health. Prev Chronic Dis 2016;13:E86.

58. Vest JR, Harle CA, Schleyer T, Dixon BE, Grannis SJ, Halverson PK, et al. Getting from here to there: health IT needs for population health. Am J Manag Care 2016;22(12):827-29.

59. King RJ, Garrett N, Kriseman J, Crum M, Rafalski EM, Sweat D, et al. A Community Health Record: Improving Health Through Multisector Collaboration, Information Sharing, and Technology. Prev Chron Dis 2016;13:E122.

60. US Department of Health and Human Services OotASfH. Public Health 3.0: a call to action to create a 21st century public health infrastructure; 2016.

61. Tabano DC, Cole E, Holve E, Davidson AJ. Distributed Data Networks That Support Public Health Information Needs. J Public Health Manag Pract 2017;23(6):674-83.

62. Sharfstein JM, Kinzer D, Colmers JM. An Update on Maryland's All-Payer Approach to Reforming the Delivery of Health Care. JAMA Intern Med 2015;175(7):1083-4.

63. CMS. Accountable Health Communities Model. Secondary Accountable Health Communities Model. https://innovation.cms.gov/initiatives/ahcm/.

64. Casey JA, Schwartz BS, Stewart WF, Adler NE. Using Electronic Health Records for Population Health Research: A Review of Methods and Applications. Annu Rev Public Health 2016;37:61-81.

65. Gutilla MJ, Davidson AJ, Daley MF, Anderson GB, Marshall JA, Magzamen S. Data for Community Health Assessment in Rural Colorado: A Comparison of Electronic Health Records to Public Health Surveys to Describe Childhood Obesity. J Public
Health Manag Pract 2017;23 Suppl 4 Supplement, Community Health Status Assessment:S53-S62.

66. Perlman SE, McVeigh KH, Thorpe LE, Jacobson L, Greene CM, Gwynn RC. Innovations in Population Health Surveillance: Using Electronic Health Records for Chronic Disease Surveillance. Am J Public Health 2017;107(6):853-57.

67. Tatem KS, Romo ML, McVeigh KH, Chan PY, Lurie-Moroni E, Thorpe LE, et al. Comparing Prevalence Estimates From Population-Based Surveys to Inform Surveillance Using Electronic Health Records. Prev Chron Dis 2017;14:E44.

68. Hatef E, Lasser EC, Kharrazi H, Perman C, Montgomery R, Weiner JP. A Population Health Measurement Framework: Evidence-Based Metrics for Assessing Community-Level Population Health in the Global Budget Context. Popul Health Manag 2017.

69. Adler NE, Glymour MM, Fielding J. Addressing Social Determinants of Health and Health Inequalities. JAMA 2016;316(16):1641-42.

70. Van Brunt D. Community Health Records: Establishing a Systematic Approach to Improving Social and Physical Determinants of Health. Am J Public Health 2017;107(3):407-12.

71. Bazemore AW, Cottrell EK, Gold R, , Hughes LS, Phillips RL, Angier H, et al. "Community vital signs": incorporating geocoded social determinants into electronic records to promote patient and population health. J Am Med Inform Assoc 2016;23(2):407-12.

72. Gottlieb L, Tobey R, Cantor J, Hessler D, Adler NE. Integrating Social And Medical Data To Improve Population Health: Opportunities And Barriers. Health Aff (Millwood) 2016;35(11):2116-23.

\section{Correspondence to:}

Roland Gamache

Center for Population Health Information Technology

Johns Hopkins Bloomberg School of Public Health

Baltimore, USA

E-mail: rgamache@pophlthinf.com 